\title{
LOSS OF PLASMA MEMBRANE LIPID ASYMMETRY CAN INDUCE ORDERED DOMAIN (RAFT) FORMATION
}

Shinako Kakuda, Guangtao Li, and Erwin London

Dept. of Biochemistry and Cell Biology

Stony Brook University

Stony Brook NY, 11794

Running Title: Plasma membrane asymmetry and domains 


\section{ABSTRACT}

The lipids in one leaflet of an asymmetric artificial lipid vesicle can induce or suppress the formation of ordered lipid domains (rafts) in the opposing leaflet. Whether suppression of domain formation might occur in plasma membranes was studied by using plasma membrane vesicles (PMVs) from RBL-2H3 cells. Ordered domain formation was assessed by FRET and fluorescence anisotropy. Ordered domains in PMV prepared by $\mathrm{N}$-ethyl maleimide (NEM) treatment formed to some extent up to about $37^{\circ} \mathrm{C}$. In contrast, ordered domains in symmetric vesicles formed from extracted PMV lipids were stable up to $55^{\circ} \mathrm{C}$. This indicates that the stability of ordered domains was substantially less in the intact PMV. A similar decrease in ordered domain stability was observed in artificial asymmetric lipid vesicles relative to the corresponding symmetric vesicles. This suggested either that the intact PMV have a significant degree of lipid asymmetry or that PMV proteins suppress domain formation. Additional experiments ruled out the latter explanation. First, stabilization of ordered domain formation relative to intact PMV was observed in protein-containing symmetric vesicles prepared by detergent solubilization of intact PMV, followed by rapid dilution of detergent. Second, ordered domain stability in intact PMV was not altered after extensively removing PMV proteins with proteinase $\mathrm{K}$. We conclude that intact NEM-induced PMV preserve a significant amount of the lipid asymmetry of plasma membranes, and that loss of PMV lipid asymmetry can induce ordered domain formation, consistent with the possibility that dynamic control of lipid asymmetry can regulate ordered domain formation in the plasma membrane.

\section{INTRODUCTION}

It is now widely accepted that the plasma membrane (PM), and likely even some internal cellular membranes, have the capacity to form co-existing liquid ordered (Lo state) lipid domains (rafts) and liquid disordered (Ld state) lipid domains [1, 2]. Ordered domain formation has even been detected in bacteria, especially those with cholesterol lipids derived from host cholesterol $[3,4]$. Most often the formation of ordered lipid domains is driven by interactions of sphingolipids and sterol, lipids rich in PM [1]. Nevertheless, much controversy remains, and how lipid raft formation, properties and size, are regulated is still unclear in most cases. Clustering of proteins with a high raft affinity is one widely studied mechanism likely to promote raft formation and enlargement [5]. However, dynamic changes in lipid asymmetry also have the potential alter domain formation [6-8]. This is because interleaflet coupling of lipid physical properties can alter raft formation when one leaflet rich in raft-forming lipids contacts a leaflet unable to spontaneously form rafts. Lipid asymmetry in which only one leaflet has the ability to form lipid rafts can result in either induction or suppression of ordered domain formation, depending upon lipid composition [6, 7, 9-11].

Light microscopy experiments have the limitation that they cannot distinguish cases in which domain formation is suppressed from cases in which large domains are converted to submicroscopic nanodomains. Fluorescence resonance energy transfer (FRET) studies can detect nanodomains, and do not have this limitation. Using FRET, we previously demonstrated that in artificial asymmetric lipid unilamellar vesicles (aLUV) whether ordered domains are induced or suppressed is very sensitive to lipid structure [6, 7]. For example, ordered domain formation is strongly suppressed in artificial asymmetric cholesterol-containing lipid vesicles mimicking $\mathrm{PM}$, with an outer leaflet containing a mixture of sphingomyelin (SM) and 1-palmitoyl 2oleoyl phosphatidylcholine (POPC), and an inner leaflet containing POPC [7]. Upon loss of asymmetry in such vesicles, induction of ordered domain formation could be detected by FRET and fluorescence anisotropy [7].

The PM has a highly asymmetric lipid distribution [12], and it has been proposed that transient loss of asymmetry may have important biological functions [13]. To investigate the persistence of asymmetry in isolated PM and whether there is a change in domain stability similar to that in artificial lipid vesicles upon loss of asymmetry, we compared domain formation in preparations of giant plasma membrane vesicles (GPMV) prepared from RBL-2H3 cells and symmetric vesicles composed of extracted PM lipids. (Note: because spectroscopic studies, unlike microscopy, report on the behavior of both the GPMV and submicroscopic 
bioRxiv preprint doi: https://doi.org/10.1101/2021.05.03.442484; this version posted May 4, 2021. The copyright holder for this preprint (which was not certified by peer review) is the author/funder, who has granted bioRxiv a license to display the preprint in perpetuity. It is made available under aCC-BY-NC-ND 4.0 International license.

vesicles present in GPMV preparations [14], we refer to the preparations used in this study as plasma membrane vesicles (PMV).)

In a previous study we demonstrated that intact PMV can form ordered domains up to physiologic temperature. In other words, they are of borderline stability at $37^{\circ} \mathrm{C}$ [14]. We now find that vesicles formed from PMV lipids have a strong ability to form ordered domains at and well above physiologic temperature. The difference in the behavior of PMV and vesicles formed from PM lipid was very similar to the difference between asymmetric and symmetric artificial lipid vesicles. Additional experiments showed that the presence of membrane associated proteins in the PMV did not explain the difference between intact PMV and vesicles formed from PMV lipids. We conclude that PMV, at least when prepared by N-ethyl maleimide (NEM) treatment, retain much of the asymmetry of intact PM, and that this asymmetry partly suppresses the ability to form ordered PM domains. Thus, there is the potential for lipid raft formation in vivo, and the consequent changes in membrane protein function, to be controlled by dynamic changes in PM lipid asymmetry.

\section{MATERIALS AND METHODS}

Materials:

Palmitoyl-2-oleoyl-sn-glycero-3-phosphocholine (POPC), egg sphingomyelin (eSM), Cholesterol (CHOL), 1,2-dioleoyl-sn-glycero-3-phosphoethanolamine- $\mathrm{N}$-(lissamine rhodamine $\mathrm{B}$ sulfonyl) (Rho-DOPE) were from Avanti Polar Lipids (Alabaster, AL). 1,6-diphenyl-1,3,5-hexatriene (DPH) was from Sigma-Aldrich (St. Louis, MO). Octydecylrhodamine B (ODRB), 3,3'-dilinoleyloxacarbocyanine perchlorate (FAST DiO), dithiothreitol (DTT), and 4 (w/v) \% paraformaldehyde (PFA) were from Invitrogen (Carlsbad, CA). All lipids and lipid probes were stored at $-20^{\circ} \mathrm{C}$. N-ethyl-maleimide (NEM) was from Sigma-Aldrich (St. Louis, MO) dissolved in methanol and stored as aliquots at $-20^{\circ} \mathrm{C}$. Proteinase $\mathrm{K}$ (ProK) was from Thermo Fisher (Bohemia, NY). Phenylmethylsulfonyl fluoride (PMSF) was from Gold Biotechnology (St. Louis, MO). Octyl glucoside was from Anatrace (Maumee, $\mathrm{OH}$ ) and dissolved in water. 10X phosphate-buffered saline (PBS), was from Bio-RAD (Hercules, CA). Dulbecco's PBS (DPBS) (200 mg/l KCl, 200 mg/l KH $\mathrm{PO}_{4}, 8 \mathrm{~g} / \mathrm{l} \mathrm{NaCl}$, and $\left.2.16 \mathrm{~g} / \mathrm{l} \mathrm{Na} 2 \mathrm{HPO}_{4}\right)$, was from Thermo Fisher Scientific (Waltham, MA). All other chemicals were reagent grade.

Cell culture

Rat basophilic leukemia (RBL-2H3) cells were a kind gift from Dr. Barbara Baird (Cornell University). Cells were grown in DMEM medium supplemented with $10 \% \mathrm{FBS}$ and $100 \mathrm{U} / \mathrm{ml}$ penicillin and streptomycin and were maintained in a humidified incubator with $5 \% \mathrm{CO}_{2}$ at $37^{\circ} \mathrm{C}$.

Preparation of giant plasma membrane vesicle (GPMV)

GPMVs were produced and isolated from RBL-2H3 cells as previously described [14, 15]. Briefly, cells grown on a $10 \mathrm{~cm}$ plate $(70-80 \%$ of confluent) were washed twice with DPBS, and then once with GPMV buffer (10 $\mathrm{mM}$ Hepes, $150 \mathrm{mM} \mathrm{NaCl}$, and $2 \mathrm{mM} \mathrm{CaCl}_{2}(\mathrm{pH} 7.4)$ ). GPMVs were produced by adding $2.5 \mathrm{ml}$ of $2 \mathrm{mM} \mathrm{NEM}$ or $2 \mathrm{mM} \mathrm{DTT} / 24 \mathrm{mM}$ PFA in GPMV buffer to the plate at $37^{\circ} \mathrm{C}$ for $2 \mathrm{~h}$. The supernatants containing GPMVs were collected, and then centrifuged at $100 \mathrm{x} \mathrm{g} / 10 \mathrm{~min}$ to remove large cell debris. The resulting PMVcontaining supernatants were used for the measurements on intact PMV in Figure 1 and 2. Because we found the GPMV induced by NEM results in contamination with small cell debris or cytosolic components that resulted in high backgrounds when measuring DPH fluorescence (see below), in Figure 1 and 2 the background intensities were subtracted for the FRET measurements. For subsequent experiments, GPMVs were pelleted by further centrifugation at $20,000 \times \mathrm{g}$ at $4^{\circ} \mathrm{C}$ for $30 \mathrm{~min}$. In this case, the background averaged around $2 \%$ of the DPH fluorescence intensity and was not subtracted from DPH fluorescence.

Phase separation detected by fluorescence microscopy

Large-scale domain formation was visualized by staining cells with Fast DiO was previously described [14]. Briefly, cells were stained with $5 \mu \mathrm{g} / \mathrm{ml}$ Fast DiO/ethanol at $4^{\circ} \mathrm{C}$ for $10 \mathrm{~min}$ and then produced PMV as above. The resulting Fast DiO labeled PMVs were pelleted by centrifugation at $500 \mathrm{x} g$ for 10 min and then suspended 
bioRxiv preprint doi: https://doi.org/10.1101/2021.05.03.442484; this version posted May 4, 2021. The copyright holder for this preprint (which was not certified by peer review) is the author/funder, who has granted bioRxiv a license to display the preprint in perpetuity. It is made available under aCC-BY-NC-ND 4.0 International license.

in 100-500 $\mu \mathrm{l}$ of GPMV buffer for microscopy imaging. The fraction of GPMV having domains were assessed in 150 GPMVs from three images as previously described.

\section{Preparation of lipid vesicles from extracted PMV lipids}

PMV pellets from two to three $10 \mathrm{~cm}$ plates were extracted by $0.8-1.2 \mathrm{ml}$ of $3: 2(\mathrm{v}: \mathrm{v})$ hexanes : isopropanol at room temperature for $30 \mathrm{~min}$. The organic solvent was dried under a nitrogen stream and hydrated with 2 $\mathrm{ml}$ of GPMV buffer for FRET measurements or about $0.2 \mathrm{ml}$ of GPMV buffer for octyl glucoside reconstitution, and proteinase $\mathrm{K}$ digestion experiments (see below). The lipid mixtures were dispersed at $70^{\circ} \mathrm{C}$ for $5 \mathrm{~min}$ and then vortexed at $55^{\circ} \mathrm{C}$ for 30 min using a multi-tube vortexer. The resulting multi-lamellar vesicles (MLVs) were subjected to seven cycles of freezing-thawing using liquid nitrogen-warm water to preparing symmetric vesicles from the PMV lipids.

Preparation of artificial symmetric large unilamellar vesicle (sLUV) and asymmetric unilamellar vesicle (aLUV)

Artificial symmetric and asymmetric lipid vesicles were prepared as previously described [6, 7]. For comparisons, symmetric vesicles were prepared similarly to asymmetric vesicles, with entrapped sucrose, and pelleted prior to use. The mol \% composition of sLUV was $22.5: 52.5: 25$ eSM : POPC : cholesterol; and of aLUV was about $45: 30: 25$ eSM : POPC : cholesterol outer leaflet and $75: 25$ POPC : cholesterol inner leaflet [7].

\section{Measurement of PMV lipid concentration in vesicles by DPH}

Lipid concentration of PMVs and artificial lipid vesicles were estimated by measuring the fluorescence intensity of membrane-inserted DPH under conditions in which DPH is in excess [14, 16]. A standard curve of bSM : POPC 1:1 SUV was prepared. To do this, $0.5 \mu \mathrm{mol} b S M$ and $0.5 \mu \mathrm{mol}$ POPC were mixed and dried under a nitrogen stream. The dried lipids were dissolved in $30 \mu \mathrm{l}$ ethanol and then $970 \mu \mathrm{l}$ of PBS was added, and incubated at $70^{\circ} \mathrm{C}$ for 5 min to disperse the lipids. Next $5 \mu \mathrm{l}$ of $0.2 \mathrm{mM} \mathrm{DPH} /$ ethanol was added to the desired dilution of the standard in PBS ( $1 \mathrm{ml}$ total volume) to give a range of $0-15 \mu \mathrm{M}$ of SUV. For the experimental samples (generally diluted to the range 0.5-3 $\mu \mathrm{M}$ ), After incubation for $5 \mathrm{~min}$ at room temperature in the dark, the intensity of DPH fluorescence in standard curve and experimental samples were measured using $\lambda e x 358$ $\mathrm{nm} ; \lambda e m 427 \mathrm{~nm}$ and slit bandpass of $3 \mathrm{~nm}$.

\section{Measurement of the temperature dependence of FRET}

FRET measurements on PMV and vesicles made from PMV lipids were performed with DPH as the FRET donor and ODRB as the FRET acceptor. Samples contained 10-16 $\mu \mathrm{M}$ lipid dispersed in $1 \mathrm{ml}$ of GPMV buffer. For "F samples" $4 \mu \mathrm{l}$ of $100 \mu \mathrm{M}$ ODRB/ethanol was then added. , "Fo samples" lacked ODRB. Both types of samples were then incubated at $37^{\circ} \mathrm{C}$ for $1 \mathrm{~h}$ in the dark. Next, $4 \mu \mathrm{l}$ of $7.5 \mu \mathrm{M} \mathrm{DPH} /$ ethanol was added to both F samples and Fo samples, and incubated at room temperature for $5 \mathrm{~min}$ in the dark. DPH fluorescence intensity was measured using a Horiba QuantaMaster Spectrofluorimeter (Horiba Scientific, Edison, NJ) using quartz semimicro cuvettes (excitation path length $10 \mathrm{~mm}$ and emission path length $4 \mathrm{~mm}$. DPH fluorescence was measured at $\lambda$ ex $358 \mathrm{~nm}$; $\lambda e m 427 \mathrm{~nm}$, and bandpass set $3 \mathrm{~nm}$ for both excitation and emission). When desired, background fluorescence intensity (i.e. in the absence of DPH) was measured from readings for both $\mathrm{F}$ samples and Fo samples. Data was collected at every $4^{\circ} \mathrm{C}$ while increasing temperature from $16^{\circ} \mathrm{C}$ to $64^{\circ} \mathrm{C}$. Between readings temperature was increased at a rate of $4^{\circ} \mathrm{C} / 5 \mathrm{~min}$. The fraction of fluorescence not quenched by FRET is equal to F/Fo. (FRET efficiency is 1-F/Fo). When necessary (data in Figures 1 and 2), background values were subtracted before F/Fo was calculated. In later experiments in which the PMV were pelleted by centrifugation to remove contaminants, the background was negligible (average about $2 \%$ of signal). F/Fo ratios were normalized to 1 at $64^{\circ} \mathrm{C}$ to compensate for variations arising from variable incorporation of ODRB into PMV. Unnormalized F/Fo data is shown in the Supplemental Materials. It should be noted the background arising from contaminants was higher in PMV prepared with NEM than those prepared with DTT/PFA, probably due to easy detachment of cells during PMV formation when using NEM. 
bioRxiv preprint doi: https://doi.org/10.1101/2021.05.03.442484; this version posted May 4, 2021. The copyright holder for this preprint (which was not certified by peer review) is the author/funder, who has granted bioRxiv a license to display the preprint in perpetuity. It is made available under aCC-BY-NC-ND 4.0 International license.

For artificial vesicles composed of SM-POPC-CHOL, the FRET measurement protocol was similar except instead of ODRB, samples contained as FRET acceptor rhodamine-DOPE (3\% of total lipid) which was mixed with the lipids in organic solvent before drying. Again, final lipid concentration was 10-16 $\mu \mathrm{M}$ dispersed in $1 \mathrm{ml}$ of PBS.

Octyl Glucoside (OG) treatment of PMV and artificial vesicles

Pellets of PMV and lipid vesicles was suspended in a small volume of PBS, to give a concentration of about 300-450 $\mu \mathrm{M}$ lipid. Then OG/water was added to give a final concentration $45 \mathrm{mM} \mathrm{OG}$. The samples were then incubated at $37^{\circ} \mathrm{C}$ for $1 \mathrm{~h}$, followed by dilution to $1.5 \mathrm{mM} \mathrm{OG}$ and $10-15 \mu \mathrm{M}$ lipid to reconstitute vesicles. For control, $1.5 \mathrm{mM}$ OG was added to PMV and lipid vesicle samples containing $10-15 \mu \mathrm{M}$ lipid.

Fluorescence anisotropy measurements

Fluorescence anisotropy was measured as previously described [7]. Lipid concentrations were 10-15 $\mu \mathrm{M}$. $\mathrm{DPH}$ was $0.03 \mu \mathrm{M}$, which gives a $\mathrm{DPH} /$ lipid ratio of $1 / 300$ to $1 / 500$.

Proteinase K (ProK) treatment

A $200 \mu \mathrm{l}$ aliquot of PMV with 100-160 $\mu \mathrm{M}$ lipid was incubated with or without $8 \mu \mathrm{g}$ of ProK in PMV buffer at $37^{\circ} \mathrm{C}$ for $1 \mathrm{~h}$ with gentle vortexing every $15 \mathrm{~min}$. ProK activity was then inhibited by adding $2 \mathrm{mM}$ phenylmethylsulfonyl fluoride (PMSF) from a stock solution in ethanol, for 15 min at room temperature. The resulting ProK treated PMVs were diluted to $2 \mathrm{ml}$ for FRET measurement as above. For checking ProK efficiency, $20 \mu$ of untreated and of ProK treated PMV (before dilution for FRET) was loaded to $8 \%$ SDSPAGE gels and the proteins were stained with GelCold Blue Stain (Thermo Fisher Scientific). A control in which PMSF was added for 15 min at room temperature before adding ProK also prepared and loaded on the gel.

\section{RESULTS}

\section{Ordered domain formation in NEM-induced and DTT/PFA-induced PMV}

We used FRET and fluorescence anisotropy to assess physical properties sensitive to ordered lipid domain formation and lipid asymmetry $[6,7,17]$. In the FRET assay, the fraction of fluorescence of the FRET donor $(\mathrm{DPH})$ unquenched by the presence of a rhodamine-containing FRET acceptor (octadecylrhodamine $\mathrm{B}$ (ODRB) or rhodamine-DOPE) $[6,14,18]$ was measured. This fraction is equal to F/Fo, where $F$ is DPH fluorescence in the presence of acceptor and Fo is DPH fluorescence in the absence of acceptor. (FRET efficiency is 1-F/Fo.) The FRET acceptors used preferentially partition into disordered domains (7), while the FRET donor DPH generally partitions nearly equally between ordered and disordered domains (18). The consequence is that FRET is weaker (F/Fo higher) in membranes containing co-existing ordered Lo domains and disordered Ld domains than it is in homogenous membranes lacking Lo domains [6, 14, 18]. At high temperatures, at which ordered domains "melt"/become miscible with Ld lipids, there is a decrease in the average distance between DPH molecules that were localized in the ordered domains and FRET acceptors that were in the disordered domains. As a result, overall F/Fo decreases. The thermal stability of ordered domains can be estimated by the temperature at the apparent midpoint (inflection point) in the roughly sigmoidal F/Fo versus temperature curves. In cases in which the transition is at too low a temperature, thermal stability can be estimated by the approximate endpoint temperature, at which the absolute value of the slope of F/Fo vs. temperature reaches a minimum [14, 19].

Fluorescence anisotropy measurements were carried out to confirm FRET results. The fluorescence anisotropy of membrane-inserted DPH, a measure of membrane order, is higher in the Lo state ( 0.3) than the Ld state ( 0.05-0.15)[20].

We first compared the temperature dependence of domain formation for PMV prepared from cultured RBL-2H3 cells by two different methods. The first is treatment of cells with a combination of dithiothreitol (DTT) and paraformaldehyde (PFA), and the second is treatment with N-ethyl maleimide (NEM)[21]. The DTT/PFA method is widely used because the large-scale domains in DTT/PFA-induced PMV can be easily 
visualized at up to almost room temperature by light microscopy, whereas large scale domains in NEMinduced PMV can be detected only at very low temperature. However, DTT/PFA is a much more perturbing chemical treatment than NEM, as it crosslinks proteins and chemically modifies phosphatidylethanolamine (PE), a major PM lipid. Comparison of nanodomain formation in NEM- and DTT/PFA-induced PMV vs. temperature, as assayed by FRET, is shown in Figure $1 \mathrm{~A}$. The nanodomains in NEM-induced PMV were significantly less thermally stable than nanodomains in DTT/PFA-induced PMV. This difference paralleled that for large scale domain formation, which disappeared above $5-10^{\circ} \mathrm{C}$ in NEM-induced PMV and $20^{\circ} \mathrm{C}$ in DTT/PFA-induced PMV (Figure 1B), roughly in agreement with previous studies [21]. As noted previously, in DTT/PFA-induced PMV nanodomain formation is much more thermally stable than large scale phase separation [14]. This Figure 1 shows this is also true for NEM-induced PMV.

We also measured the anisotropy of NEM- and DTT/PFA-induced PMV at $16^{\circ} \mathrm{C}$ and $64^{\circ} \mathrm{C}$ (Figure $1 \mathrm{C}$ ). Both preparations exhibited high anisotropy at low temperature, consistent with a high level of ordered domain formation, and low anisotropy indicative of Ld state at high temperature, indicating loss of ordered domain formation at high temperature. The difference between ordered domain stability in the two preparations does not seem to reflect a lower amount of SM relative to PC in the NEM-induced PMV relative to that in the DTT/PFA-induced PMV (Supplemental Figure 1B and 1C). A difference in asymmetry might be responsible for the difference in ordered domain thermal stability (see below). For all subsequent experiments, the less chemically perturbed NEM-induced PMV were used.

\section{Ordered domain formation in PMV is enhanced in vesicles prepared from PMV lipid extracts}

We recently reported that loss of lipid asymmetry induced ordered lipid domain formation in artificial vesicles that roughly mimic PM [7]. Therefore, we hypothesized that if PMV retained a significant level of asymmetry, symmetric lipid vesicles composed of PMV lipids would form more stable ordered domains than those in intact PMV. To test this hypothesis, we prepared artificial large unilamellar vesicles (which exhibit lipid symmetry [22-24] ) from extracted PMV lipids, and compared ordered domain stability in these vesicles to that in intact PMV. As predicted, the FRET curve demonstrated that, ordered domains formed in PMV lipid were considerably more thermally stable, persisting to $55^{\circ} \mathrm{C}$, than those in intact PMV, which disappeared above about $37^{\circ} \mathrm{C}$ (Figure 2A). These results were confirmed by DPH fluorescence anisotropy (Figure 2B).

Anisotropy vs. temperature curve closely paralleled the FRET curve for PMV lipid, with the highly ordered state being lost above $55^{\circ} \mathrm{C}$. Note that a weaker temperature dependent decrease in anisotropy continues above the temperature at which the domains melt. This decrease occurs because a disordered state bilayer becomes increasingly disordered as temperature is increased. Anisotropy in intact PMV also showed a domain melting event in which ordered domains disappeared (at about $37^{\circ} \mathrm{C}$ ), similar to that detected by FRET. It is noteworthy that at low temperature DPH anisotropy in intact PMV was lower than that for PMV lipid vesicles (Figure 2B). This suggests a lower extent of ordered domain formation in intact PMV than in PMV lipid at low temperature. Overall, the conclusions from FRET and anisotropy are in good agreement.

\section{A loss of asymmetry explains suppression of ordered domain formation in PMV}

The results described above strongly suggest that, as is the case in artificial vesicles with a simple lipid composition [6, 7], the loss of asymmetry in PM can induce/enhance ordered domain formation. However, another possibility is that proteins associated with intact PMV are responsible for the difference in behavior of intact PMV and that of vesicles formed from extracted PMV lipids. To examine this possibility, we first determined whether treating intact PMV under conditions that should destroy asymmetry, but not remove membrane proteins, would destabilize ordered domain formation. Vesicles were dissolved in detergent and then the detergent was diluted to a concentration well below its critical micelle concentration to form vesicles $[25,26]$.

To confirm that detergent treatment destroyed asymmetry, we first studied the effect of detergent solubilization and dilution using octyl glucoside $(\mathrm{OG})$ upon asymmetric artificial lipid vesicles that crudely mimic PM. The behavior of asymmetric unilamellar vesicles (aLUV) with SM and POPC in the outer leaflet, POPC in 
the inner leaflet and cholesterol in both leaflets, was compared to that of ordinary symmetric unilamellar vesicles (sLUV) composed of SM-POPC-cholesterol. As shown in Figure 3A FRET measurements showed that for the aLUV after OG solubilization and reconstitution ordered domain formation was increased and thermally stabilized relative to untreated samples, in which prior studies found there is little, if any, domain formation [7]. Control experiments simply adding OG to aLUV at the final diluted OG concentration (OG control) showed little effect on FRET. Figure 3B shows that there was also little effect on ordered domain formation and stability when the symmetric SM-POPC-cholesterol vesicles (with about the same overall composition as the aLUV) were first dissolved in OG and then vesicles reconstituted by dilution, as expected as vesicles should remain symmetric after reconstitution. Therefore, stabilization of ordered domain formation upon solubilization and reconstitution is specific to the asymmetric vesicles. This is consistent with our previous report showing that a loss of asymmetry triggered by heating vesicles can induce ordered domain formation in aLUV composed of SM, POPC and cholesterol [7].

Analogous experiments were then carried out upon intact PMV and PMV lipid (Figures 3C and 3D). FRET showed that reconstitution of PMV after solubilization with OG increased ordered domain stability to a level similar to that in the vesicles composed of PMV lipids. This was not due to a loss of protein upon solubilization and reconstitution. Sucrose gradient centrifugation on OG reconstituted vesicles formed from intact PMV was compared to that for OG reconstituted vesicles formed from PMV lipids. As shown in Supplemental Figure 4, the vesicles formed from PMV had a higher density than the vesicles from just PMV lipids, and protein co-migrated with lipid in the PMV-derived samples. These results imply that the change in ordered domain stability was not due to loss of protein, but to a loss of asymmetry. The fact that domain stability was not alter in controls in which OG was added at the final diluted concentration (Figure 3C) indicates $O G$ itself was not responsible for the increase in ordered domain formation. The observation that there was almost no effect upon ordered domain stability after reconstituting OG-solubilized vesicles composed of PMV lipid (Figure 3D), which should already be symmetric before OG reconstitution, also indicates that a loss of asymmetry had occurred after OG reconstitution of intact PMV.

The conclusion that upon reconstitution of OG-solubilized artificial aLUV there was increased ordered domain stability associated with a loss of asymmetry was confirmed by DPH fluorescence anisotropy. Figure 4A shows that untreated SM-POPC-cholesterol SLUV containing ordered domains exhibited a high degree of anisotropy at low temperature $\left(16^{\circ} \mathrm{C}\right)$, and OG reconstitution of sLUV had no significant effect on membrane order (Figure 4A, left). In contrast, untreated aLUV with about the same overall lipid composition as the sLUV had a much lower level of anisotropy than untreated sLUV (Figure 4A, right), consistent with the FRET result indicating very little ordered domain formation in aLUV. However, after solubilization and reconstitution the anisotropy levels of aLUV increased to that found in sLUV (Figure 4A, left). Adding OG directly to aLUV at the final dilute concentration present after reconstitution has little effect on anisotropy (Figure 4C, right). These experiments confirm the FRET results, showing that OG solubilization and dilution to destroy asymmetry in aLUV induces domain formation.

Figure 4B shows analogous experiments for vesicles formed from PMV lipid and intact PMV. Vesicles from PMV lipid, and those formed after OG solubilization and reconstitution of those vesicles, both exhibited a similar high degree of anisotropy (Figure 4B, left). In contrast, intact PMV had a lower level of anisotropy than that of the vesicles formed after solubilization and reconstitution of PMV (Figure 4B right). In the vesicles from intact PMV formed by reconstitution after solubilization with $O G$, anisotropy levels increased to that found in vesicles formed from PMV lipids. This was true even though the vesicles reconstituted after solubilization of intact PMV contain PMV proteins, as noted above. Again, addition of OG to PMV at the final dilute concentration after reconstitution had little effect on anisotropy (Figure 4D, right). Thus, the effect of vesicle solubilization and reconstitution upon DPH fluorescence anisotropy in intact PMV and vesicles formed from PMV lipid agrees with the FRET results, as well as the anisotropy changes seen after reconstitution of artificial asymmetric and symmetric lipid vesicles, respectively.

To further investigate if PMV proteins suppress ordered domain formation in intact PMV, PMVs were treated with proteinase $\mathrm{K}$ (ProK), a non-specific protease that strongly degrades proteins. There was no increase in the thermal stability of ordered domain formation as assayed by FRET (Figure 5A) and no large 
change in DPH fluorescence anisotropy after digestion with ProK (Figure 5B) despite extensive degradation of proteins (Figure 5C). If there was any significant change, ProK treatment appeared to reduce, not to increase, membrane order slightly, at least at elevated temperature.

Thus, both FRET and anisotropy data strongly supports the conclusion that intact PMV have suppressed ordered domain formation largely due to their lipid asymmetry, and that loss of this asymmetry stabilizes ordered domain formation. Based on both the reconstitution and ProK treatment results, it is unlikely that proteins are the main reason that raft formation in intact PMV is suppressed relative to that in vesicles formed from PMV lipids. However, this does not prove proteins have no effect on ordered domain formation (see Discussion).

\section{DISCUSSION}

\section{Capacity of PMV and PM lipids to form ordered membrane domains and dependence upon lipid asymmetry}

For this reason, we measured physical properties that assess domain formation and membrane order, which several studies have shown is sensitive to asymmetry $[6,7,17,27]$. The studies show that PM lipids in symmetric lipid vesicles have a strong ability to form co-existing ordered and disordered lipid domains. The ordered domains were stable to well above physiologic temperature. In contrast, intact PMV formed ordered domains with much lower thermal stability. Based on prior studies that show asymmetry can repress ordered domain formation [6, 7], this suggested that intact PMV have considerable lipid asymmetry. This was confirmed by the observations that: 1. detergent reconstitution of PMV to destroy asymmetry increased ordered domain stability to levels seen in symmetric lipid vesicles, and 2. PMV and symmetric vesicles formed from PMV lipids exhibited properties and differences in terms of domain formation, membrane order, and responses to detergent reconstitution, that were very similar to the properties of, and differences between, asymmetric and symmetric SM-POPC-cholesterol lipid vesicles for these parameters. The observation that ordered domains formed by DTT/PFA-induced PMV are more thermally stable than those by NEM-induced PMV, even though the two preparations have similar SM/PC ratios (Supplemental Figure 1C), may indicate that DTT/PFA-induced PMV may have lost more asymmetry than NEM-induced PMV.

Although the similarity between PMV and artificial asymmetric vesicles indicates PMV have lipid asymmetry, they do not reveal the exact extent of PMV asymmetry. Loss of some degree of phosphatidylserine (PS) asymmetry is associated with GPMV production [28]. Unfortunately, because the large majority of PMV are permeable to proteins $[13,29]$, PMV asymmetry cannot readily be assessed by methods such as phospholipase digestion or chemical labeling [23, 30].

\section{Potential Implications of changes in asymmetry for ordered domain formation in vivo.}

The observation that loss of PM asymmetry could induce formation of stable ordered domains under physiologic conditions at which they would not otherwise form, or would be of only borderline stability, is of potential biological significance because transient loss of asymmetry in PM is associated with a number of biological events, including signal transduction [8]. In vivo, it is likely that activation of lipid scramblases, such as TMEM16F, are responsible for the loss of lipid asymmetry [31]. TMEM16F is activated by cytosolic $\mathrm{Ca}^{2+}$, and cytosolic $\mathrm{Ca}^{2+}$ concentration becomes elevated during signal transduction [31]. This could induce ordered domain formation. It is also possible that ordered domains formation could be induced in PM prior to loss of asymmetry, upon clustering of proteins that favor formation of Lo domains, or upon changes in PM interactions with cytoskeletal proteins. In that case, the loss of lipid asymmetry might be part of a positive feedback loop, in which receptor activation and clustering induces or enhances ordered domain formation, followed by increases in cytosolic $\mathrm{Ca}^{2+}$ that activate lipid scramblases, resulting in a loss of asymmetry that further enhances ordered domain formation.

\section{Possible effect of proteins upon ordered domain formation}


bioRxiv preprint doi: https://doi.org/10.1101/2021.05.03.442484; this version posted May 4, 2021. The copyright holder for this preprint (which was not certified by peer review) is the author/funder, who has granted bioRxiv a license to display the preprint in perpetuity. It is made

Our studies do not rule out the possibility that proteins have biophysical effects that modulate ordered domain formation. Overall, TM segments in PM proteins have amino acid sequences in contact with the outer leaflet lipids that favor contact with Lo state lipid, and sequences in contact with the inner leaflet lipids that favor interaction with Ld state lipid [32]. This raises the possibility that TM segments could influence PM lipid physical state, including their propensity to form ordered domains. It should also be noted with regard to our experiments that proteinase $\mathrm{K}$ does not digest all TM segments of membrane proteins, so there could be residual protein segments in the PMV after digestion [33], and these could alter ordered domain formation. However, at least at high temperature, proteinase $\mathrm{K}$ digestion seemed to reduce, not increase, membrane order, the opposite of what is expected the effect of protein is to inhibit ordered domain formation.

\section{Implications for interpretation of detergent resistant membrane formation from cells}

It is known that lipids in the Lo state can be detergent insoluble, and that the detergent resistant membranes (DRM) isolated from cells [34] are at least largely in the Lo state [32, 35, 36]. However, DRM are not likely to be identical to the ordered domains present in cells in the absence of detergent. The most widely used protocols involve incubation with Triton X-100 at $4^{\circ} \mathrm{C}$, and low temperature could induce the formation of ordered domains not present at physiological temperature [1]. In addition, based on studies in artificial symmetric lipid vesicles, it has been proposed that the Triton X-100 bound to membranes can induce lateral segregation of Lo and Ld domains [37], although our prior studies indicate instead that Triton X-100 only induces coalescence of preexisting ordered nanodomains [18]. In any case, the present studies suggest that a loss of asymmetry could be additional issue that must be considered. Because detergent can induce lipid flipflop, which would destroy asymmetry [38, 39], a detergent-induced loss of lipid asymmetry might enhance DRM formation upon detergent treatment of cells.

Acknowledgement: This work was supported by NIH grant GM 122493 to E.L. 
bioRxiv preprint doi: https://doi.org/10.1101/2021.05.03.442484; this version posted May 4, 2021. The copyright holder for this preprint (which was not certified by peer review) is the author/funder, who has granted bioRxiv a license to display the preprint in perpetuity. It is made available under aCC-BY-NC-ND 4.0 International license.

\section{References}

[1] D.A. Brown, E. London, Structure and function of sphingolipid- and cholesterol-rich membrane rafts, J Biol Chem 275(23) (2000) 17221-4.

[2] H.Y. Wang, D. Bharti, I. Levental, Membrane Heterogeneity Beyond the Plasma Membrane, Front Cell Dev Biol 8 (2020) 580814.

[3] T.J. LaRocca, J.T. Crowley, B.J. Cusack, P. Pathak, J. Benach, E. London, J.C. Garcia-Monco, J.L. Benach, Cholesterol lipids of Borrelia burgdorferi form lipid rafts and are required for the bactericidal activity of a complement-independent antibody, Cell Host Microbe 8(4) (2010) 331-42.

[4] T.J. LaRocca, P. Pathak, S. Chiantia, A. Toledo, J.R. Silvius, J.L. Benach, E. London, Proving lipid rafts exist: membrane domains in the prokaryote Borrelia burgdorferi have the same properties as eukaryotic lipid rafts, PLoS Pathog 9(5) (2013) e1003353.

[5] S. Schuck, K. Simons, Polarized sorting in epithelial cells: raft clustering and the biogenesis of the apical membrane, J Cell Sci 117(Pt 25) (2004) 5955-64.

[6] Q. Wang, E. London, Lipid Structure and Composition Control Consequences of Interleaflet Coupling in Asymmetric Vesicles, Biophys J 115(4) (2018) 664-678.

[7] J.W. St Clair, S. Kakuda, E. London, Induction of Ordered Lipid Raft Domain Formation by Loss of Lipid Asymmetry, Biophys J 119(3) (2020) 483-492.

[8] M. Doktorova, J.L. Symons, I. Levental, Structural and functional consequences of reversible lipid asymmetry in living membranes, Nat Chem Biol 16(12) (2020) 1321-1330.

[9] Q. Lin, E. London, Ordered raft domains induced by outer leaflet sphingomyelin in cholesterol-rich asymmetric vesicles, Biophys J 108(9) (2015) 2212-22.

[10] M.D. Collins, S.L. Keller, Tuning lipid mixtures to induce or suppress domain formation across leaflets of unsupported asymmetric bilayers, Proceedings of the National Academy of Sciences 105(1) (2008) 124-128.

[11] C. Wan, V. Kiessling, L.K. Tamm, Coupling of cholesterol-rich lipid phases in asymmetric bilayers, Biochemistry 47(7) (2008) 2190-2198.

[12] A. Verkleij, R. Zwaal, B. Roelofsen, P. Comfurius, D. Kastelijn, L. Van Deenen, The asymmetric distribution of phospholipids in the human red cell membrane. A combined study using phospholipases and freeze-etch electron microscopy, Biochimica et Biophysica Acta (BBA)-Biomembranes 323(2) (1973) 178-193. [13] A.D. Skinkle, K.R. Levental, I. Levental, Cell-Derived Plasma Membrane Vesicles Are Permeable to Hydrophilic Macromolecules, Biophys J 118(6) (2020) 1292-1300.

[14] G. Li, Q. Wang, S. Kakuda, E. London, Nanodomains can persist at physiologic temperature in plasma membrane vesicles and be modulated by altering cell lipids, J Lipid Res 61(5) (2020) 758-766.

[15] E. Sezgin, H.J. Kaiser, T. Baumgart, P. Schwille, K. Simons, I. Levental, Elucidating membrane structure and protein behavior using giant plasma membrane vesicles, Nat Protoc 7(6) (2012) 1042-51.

[16] E. London, G.W. Feigenson, A convenient and sensitive fluorescence assay for phospholipid vesicles using diphenylhexatriene, Anal Biochem 88(1) (1978) 203-11.

[17] H.T. Cheng, Megha, E. London, Preparation and properties of asymmetric vesicles that mimic cell membranes: effect upon lipid raft formation and transmembrane helix orientation, J Biol Chem 284(10) (2009) 6079-92.

[18] P. Pathak, E. London, Measurement of lipid nanodomain (raft) formation and size in sphingomyelin/POPC/cholesterol vesicles shows TX-100 and transmembrane helices increase domain size by coalescing preexisting nanodomains but do not induce domain formation, Biophys J 101(10) (2011) 2417-25. [19] J.W. St Clair, E. London, Effect of sterol structure on ordered membrane domain (raft) stability in symmetric and asymmetric vesicles, Biochim Biophys Acta Biomembr 1861(6) (2019) 1112-1122.

[20] O. Bakht, P. Pathak, E. London, Effect of the structure of lipids favoring disordered domain formation on the stability of cholesterol-containing ordered domains (lipid rafts): identification of multiple raft-stabilization mechanisms, Biophys J 93(12) (2007) 4307-18.

[21] I. Levental, M. Grzybek, K. Simons, Raft domains of variable properties and compositions in plasma membrane vesicles, Proc Natl Acad Sci U S A 108(28) (2011) 11411-6.

[22] A. Kumar, C.M. Gupta, Transbilayer distributions of red cell membrane phospholipids in unilamellar vesicles, Biochim Biophys Acta 769(2) (1984) 419-28.

[23] M.J. Hope, P.R. Cullis, Lipid asymmetry induced by transmembrane pH gradients in large unilamellar vesicles, J Biol Chem 262(9) (1987) 4360-6. 
bioRxiv preprint doi: https://doi.org/10.1101/2021.05.03.442484; this version posted May 4, 2021. The copyright holder for this preprint (which was not certified by peer review) is the author/funder, who has granted bioRxiv a license to display the preprint in perpetuity. It is made available under aCC-BY-NC-ND 4.0 International license.

[24] M.J. Hope, T.E. Redelmeier, K.F. Wong, W. Rodrigueza, P.R. Cullis, Phospholipid asymmetry in large unilamellar vesicles induced by transmembrane pH gradients, Biochemistry 28(10) (1989) 4181-7.

[25] M.L. Jackson, B.J. Litman, Rhodopsin-egg phosphatidylcholine reconstitution by an octyl glucoside dilution procedure, Biochim Biophys Acta 812(2) (1985) 369-76.

[26] Q. Mao, T. Schunk, K. Flukiger, B. Erni, Functional reconstitution of the purified mannose phosphotransferase system of Escherichia coli into phospholipid vesicles, J Biol Chem 270(10) (1995) 525865.

[27] M. Son, E. London, The dependence of lipid asymmetry upon phosphatidylcholine acyl chain structure, J Lipid Res 54(1) (2013) 223-31.

[28] H. Keller, M. Lorizate, P. Schwille, $\mathrm{Pl}(4,5) \mathrm{P} 2$ degradation promotes the formation of cytoskeleton-free model membrane systems, Chemphyschem 10(16) (2009) 2805-12.

[29] S. Zemljic Jokhadar, U. Klancnik, M. Grundner, T. Svelc Kebe, S. Vrhovec Hartman, M. Liovic, J. Derganc, GPMVs in variable physiological conditions: could they be used for therapy delivery?, BMC Biophys 11 (2018) 1.

[30] J.H. Lorent, K.R. Levental, L. Ganesan, G. Rivera-Longsworth, E. Sezgin, M. Doktorova, E. Lyman, I. Levental, Plasma membranes are asymmetric in lipid unsaturation, packing and protein shape, Nat Chem Biol 16(6) (2020) 644-652.

[31] J. Suzuki, M. Umeda, P.J. Sims, S. Nagata, Calcium-dependent phospholipid scrambling by TMEM16F, Nature 468(7325) (2010) 834-8.

[32] H.J. Sharpe, T.J. Stevens, S. Munro, A comprehensive comparison of transmembrane domains reveals organelle-specific properties, Cell 142(1) (2010) 158-69.

[33] M.E. Dumont, J. Trewhella, D.M. Engelman, F.M. Richards, Stability of transmembrane regions in bacteriorhodopsin studied by progressive proteolysis, J Membr Biol 88(3) (1985) 233-47.

[34] D.A. Brown, J.K. Rose, Sorting of GPI-anchored proteins to glycolipid-enriched membrane subdomains during transport to the apical cell surface, Cell 68(3) (1992) 533-44.

[35] R. Schroeder, E. London, D. Brown, Interactions between saturated acyl chains confer detergent resistance on lipids and glycosylphosphatidylinositol (GPI)-anchored proteins: GPI-anchored proteins in liposomes and cells show similar behavior, Proc Natl Acad Sci U S A 91(25) (1994) 12130-4.

[36] S.N. Ahmed, D.A. Brown, E. London, On the origin of sphingolipid/cholesterol-rich detergent-insoluble cell membranes: physiological concentrations of cholesterol and sphingolipid induce formation of a detergentinsoluble, liquid-ordered lipid phase in model membranes, Biochemistry 36(36) (1997) 10944-53.

[37] H. Heerklotz, Triton promotes domain formation in lipid raft mixtures, Biophys J 83(5) (2002) 2693-701. [38] L. Dietel, L. Kalie, H. Heerklotz, Lipid Scrambling Induced by Membrane-Active Substances, Biophys J 119(4) (2020) 767-779.

[39] E. Pantaler, D. Kamp, C.W. Haest, Acceleration of phospholipid flip-flop in the erythrocyte membrane by detergents differing in polar head group and alkyl chain length, Biochim Biophys Acta 1509(1-2) (2000) 397408. 

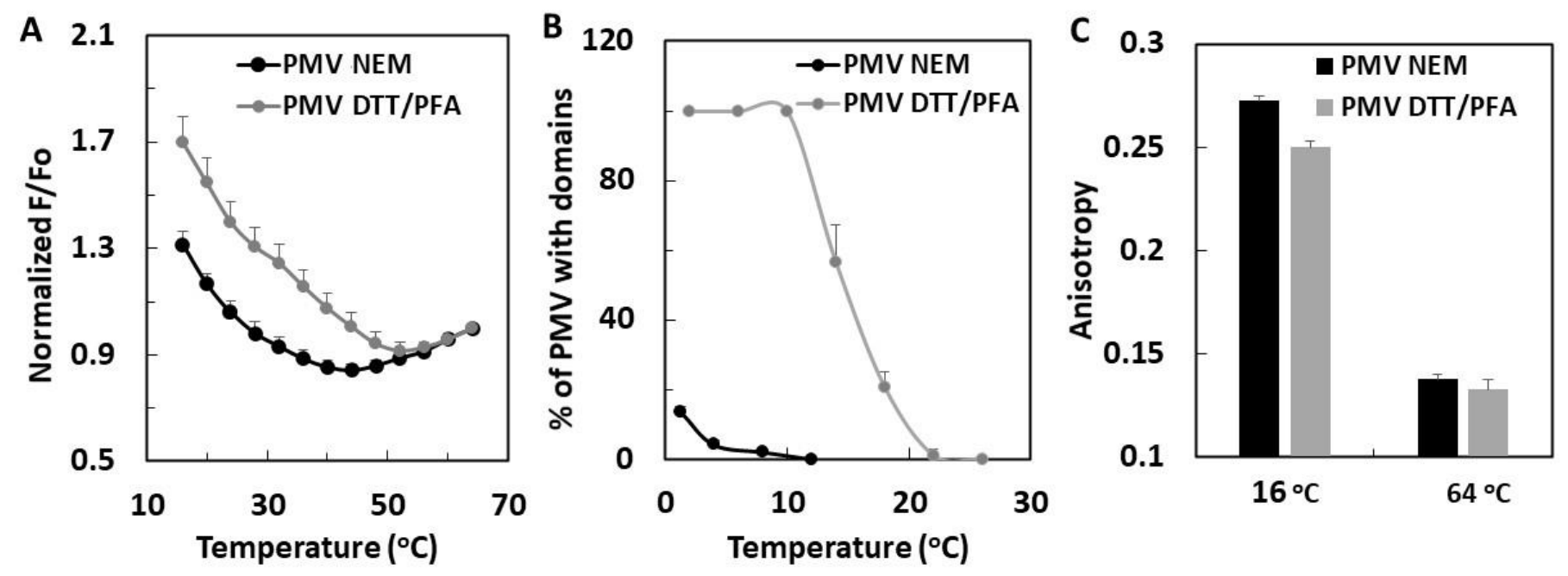

Figure 1. Temperature dependence of ordered domain formation in PMV from RBL-2H3 cells. (A) Nanodomain formation detected by FRET. DPH (FRET donor) and ODRB (FRET acceptor) were added to $F$ samples. Only DPH added to Fo samples. F/Fo values normalized to 1 at $64^{\circ} \mathrm{C}$. (B) Phase separation detected by fluorescence microscopy. Cells labeled with FAST DiO before induction of PMV. (C) Fluorescence anisotropy of membrane-inserted DPH. Gray lines and bars are PMV prepared by DTT-PFA, black lines and bars are PMV prepared by NEM. Unless otherwise noted, in this and following figures mean values and standard deviations (only up direction bar is shown) from three independent preparations are shown. For this and following figures unnormalized F/Fo vs. temperature FRET curves are shown in Supplemental Figures. 

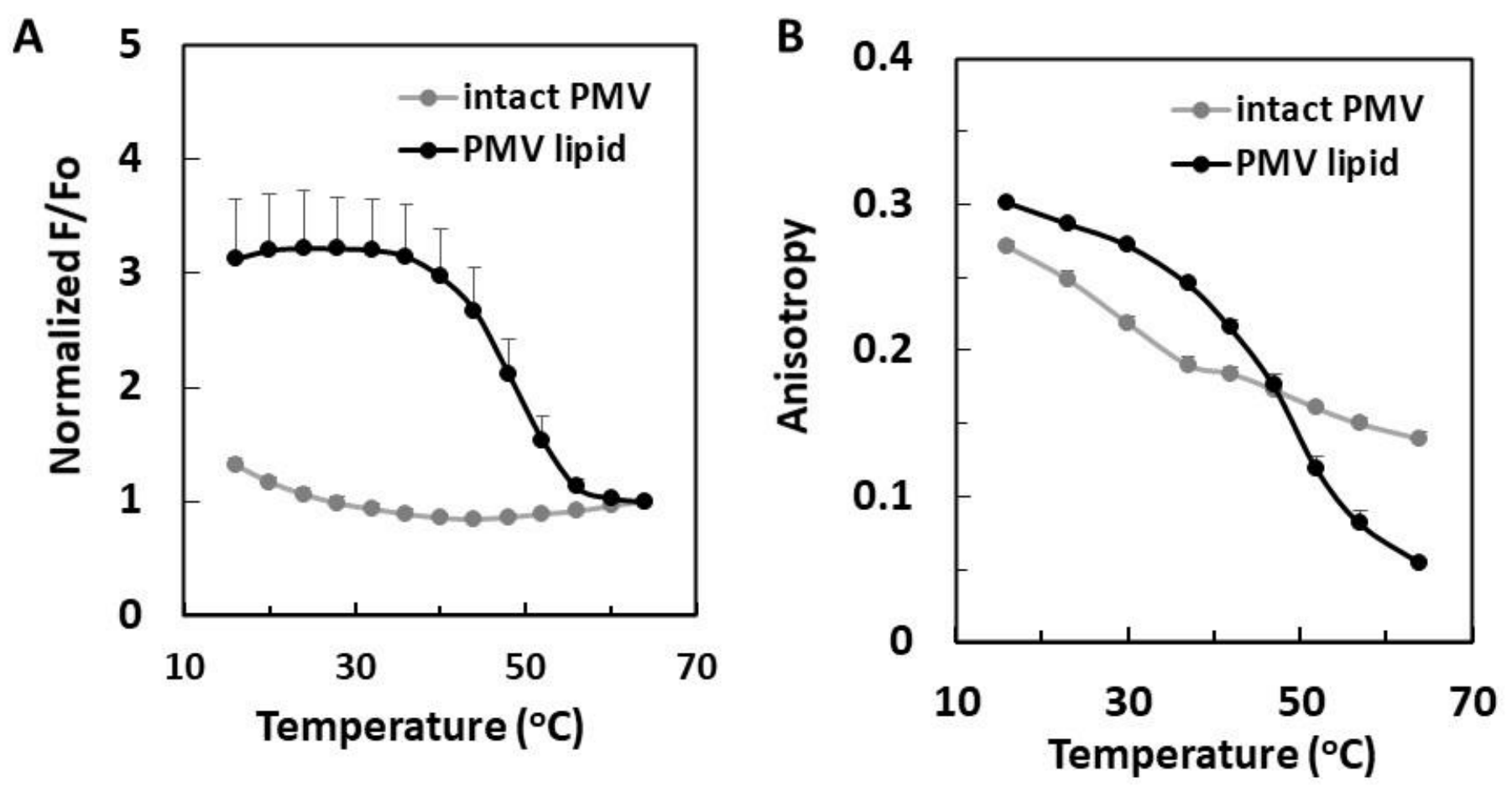

Figure 2. Comparison of domain formation and membrane order in intact PMV and lipid vesicles prepared from extracted PMV lipids. In this and subsequent figures PMV prepared using the NEM method. (A) Temperature dependence of nanodomain formation detected by FRET. (B) Temperature dependence of membrane order by fluorescence anisotropy of membrane-inserted DPH: (gray line) intact PMV, (black line) lipid vesicles extracted from intact PMV. 
A

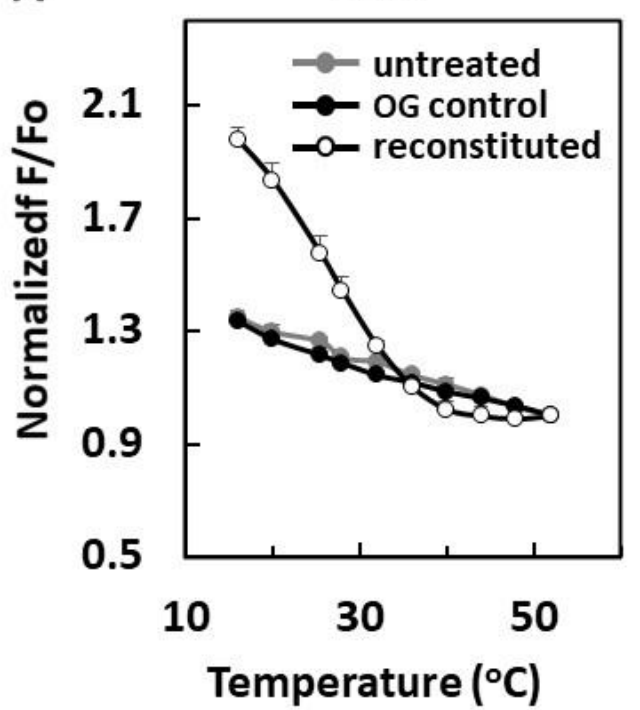

B

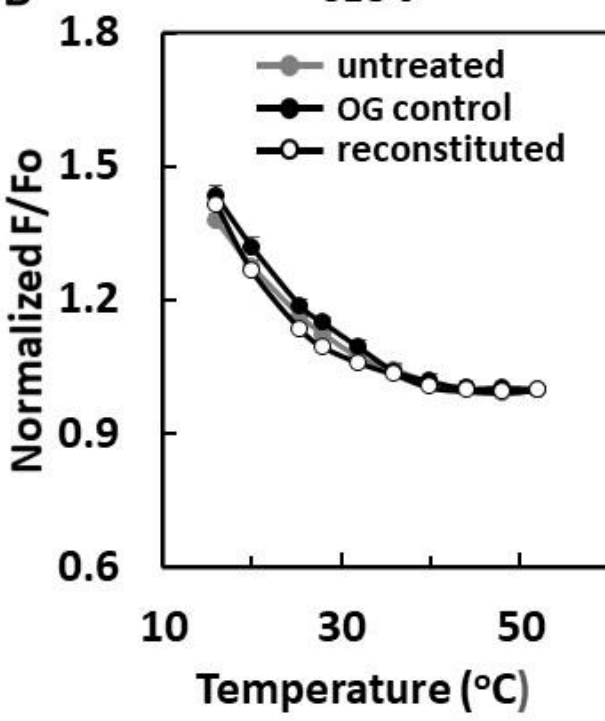

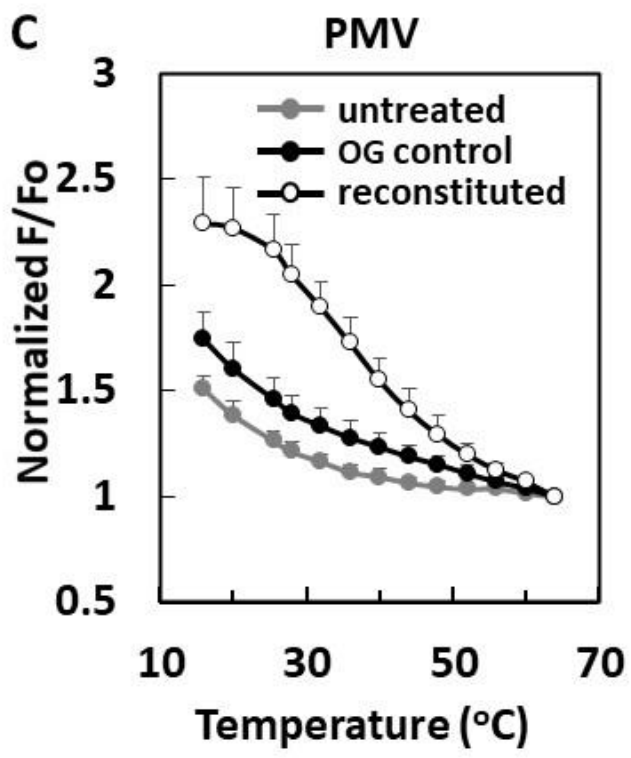

D

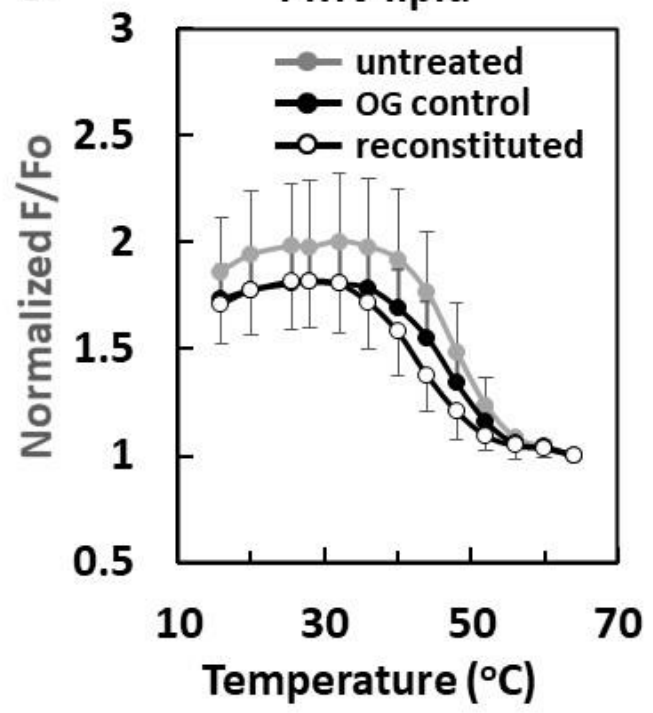

Figure 3. Effect of OG reconstituted membranes upon domain formation. Samples contained: (A) aLUV of SM-POPC outside/POPC inside/cholesterol (see the composition ratio in methods); (B) SLUV of SM-POPCcholesterol (see the composition in methods); (C) intact PMV; (D) PMV lipids. FRET in vesicles PMV derived samples used DPH as donor and ODRB as acceptor. FRET in artificial LUV used DPH as donor and rhodamine-DOPE as acceptor. FRET measured on: (gray circles) untreated vesicles; (open circles) reconstituted vesicles prepared by dissolving vesicles in $45 \mathrm{mM} \mathrm{OG}$ and then diluting to $1.5 \mathrm{mM} \mathrm{OG}$; (black circles) vesicles to which $1.5 \mathrm{mM}$ OG was added as a control (OG control). For clarity, in 3D. standard deviations for reconstituted vesicles are illustrated as down bars. 

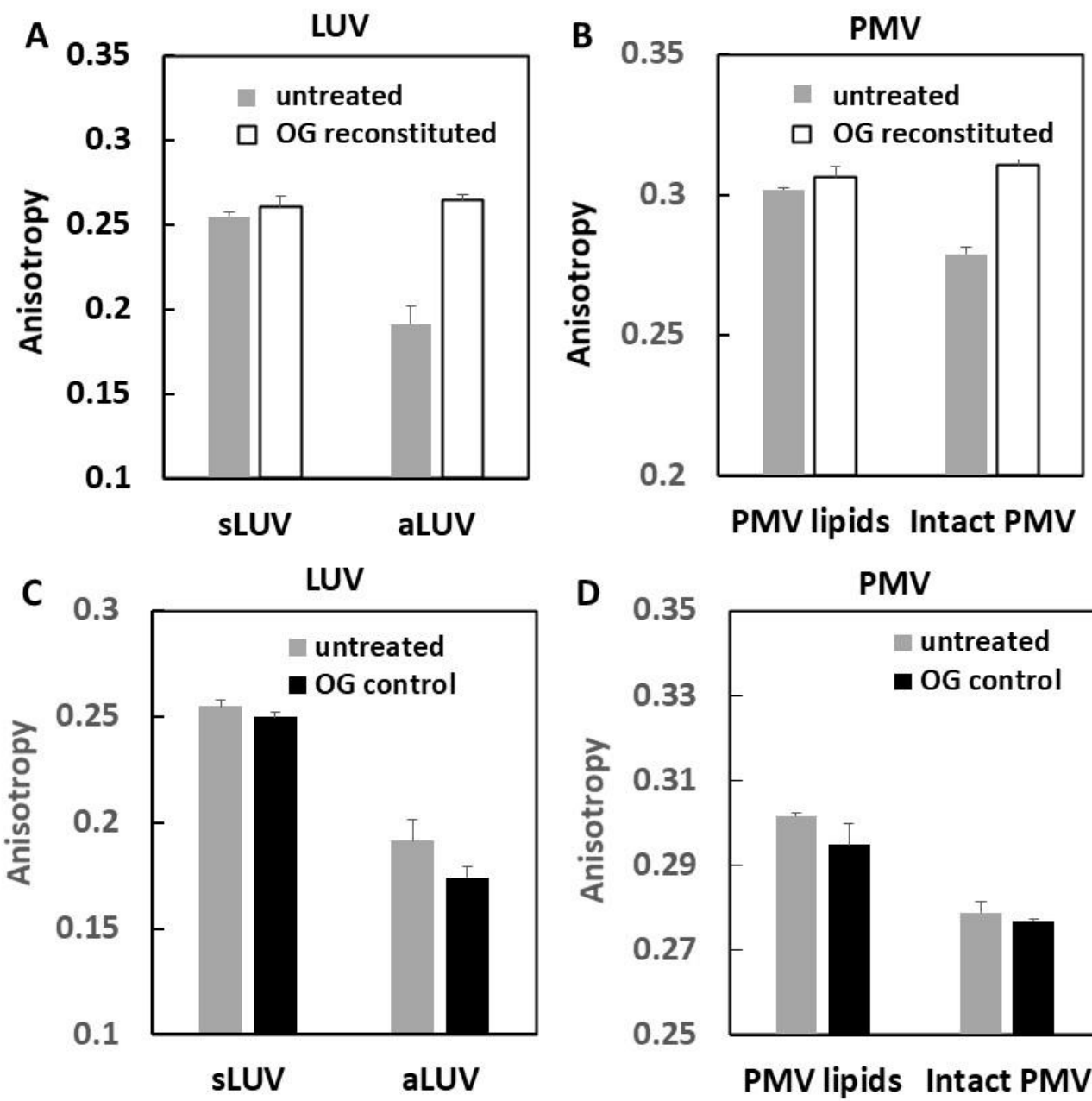

PMV lipids Intact PMV

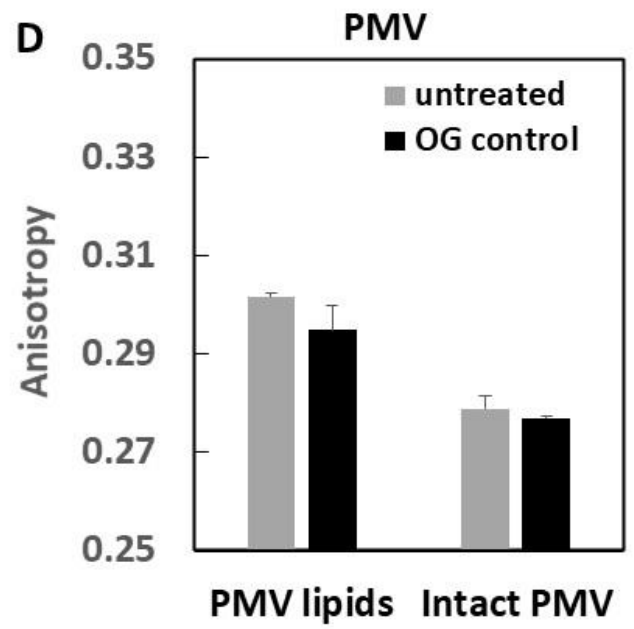

Figure 4 Reconstitution of intact PMV increases membrane order. (A and C) Artificial LUV, (B and D) PMV derived vesicles. Anisotropy of DPH fluorescence was measured at $16^{\circ} \mathrm{C}$ on: (gray bars) untreated vesicles; (open bars) reconstituted vesicles prepared by dissolving lipid vesicles in $45 \mathrm{mM} \mathrm{OG}$ and then diluting to 1.5 mM OG; (black bars) vesicles to which 1.5 mM OG was added as a control (OG control). 

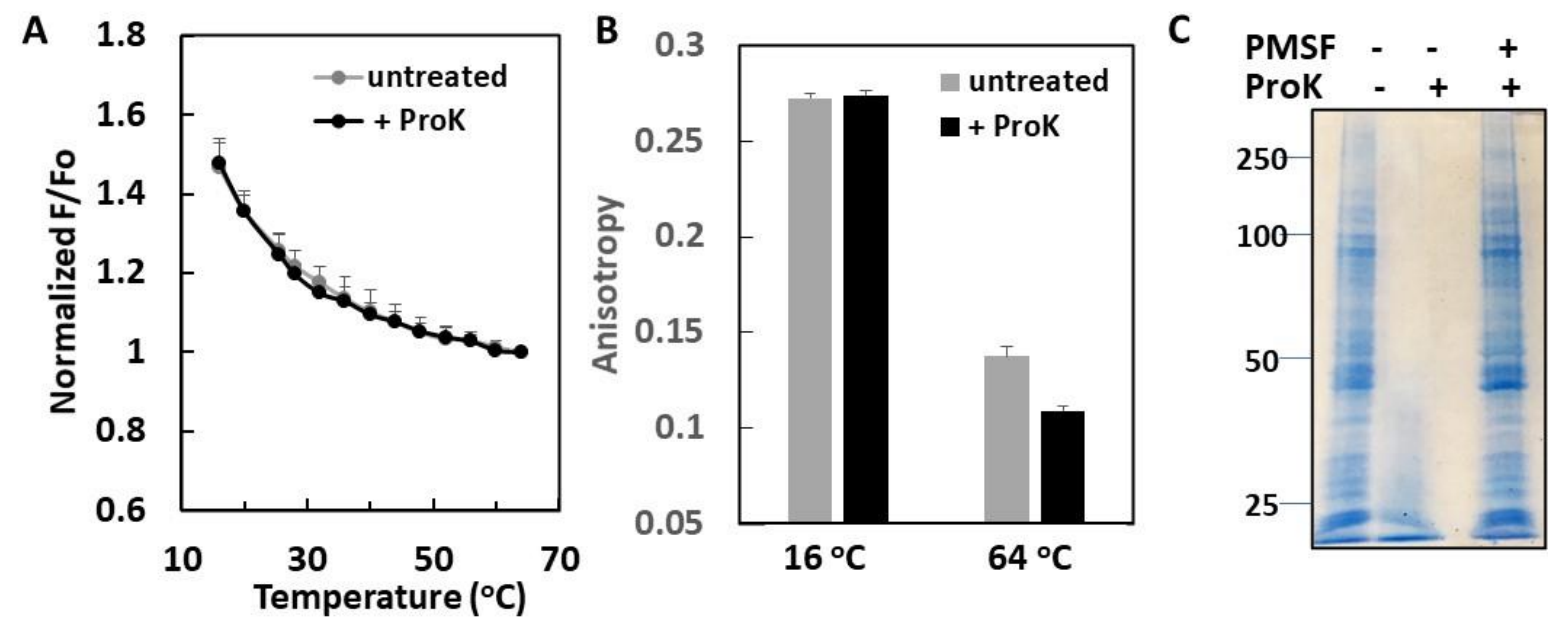

Figure 5. Effect of ProK treatment of intact PMV upon domain formation. (A) FRET measurements of (gray line) untreated and (black line) ProK treated intact PMV. FRET donor was DPH and acceptor was ODRB. (B) Anisotropy of (gray bars) untreated and (back bars) ProK treated intact PMV at $16{ }^{\circ} \mathrm{C}$ and $64^{\circ} \mathrm{C}$. (C) Example of SDS-PAGE analysis of the efficiency of ProK treatment. (left lane) untreated, (middle lane) ProK treated, (right lane) control for ProK treatment 